\title{
A radial heat flow apparatus for thermal conductivity characterisation of cylindrical samples
}

\author{
Loïc Fave $^{1}\left(\mathbb{D} \cdot\right.$ Manuel A. Pouchon $^{1} \cdot$ Cécile Hébert $^{2}$
}

Received: 19 October 2016/Accepted: 13 July 2017/Published online: 31 July 2017

(C) Akadémiai Kiadó, Budapest, Hungary 2017

\begin{abstract}
This paper presents a recent implementation of the radial heat flow method, a classical technique used to measure the thermal conductivity of materials, including the hardware designed to this end, as well as the theoretical formalism used to analyse the data. The novelty is in its application to high-aspect-ratio hollow cylindrical samples, more specifically sections of nuclear cladding tube, whereby samples representative of full length fuel clads can be measured instead of platelets, like in usual laserflash measurements. The apparatus designed and built to this end can accommodate tubular samples of varying length, diameter and wall thicknesses made of most materials, with the specific goal of determining the through-thickness thermal conductivity of silicon carbide composites, a highly anisotropic material under consideration as a fuel cladding material.
\end{abstract}

Keywords Thermal conductivity - Thermal radiation . Radial heat flow - Effective thermal conductivity . Composite materials $\cdot$ Steady-state methods

\section{Introduction}

Currently, there are significant efforts in nuclear materials research aiming at the development of new structural materials, which would better cope with

Loïc Fave

loic.fave@alumni.epfl.ch

1 Laboratory for Nuclear Materials, Paul Scherrer Institut, 5232 Villigen, Switzerland

2 Centre Interdisciplinaire de Microscopie Électronique, École Polytechnique Fédérale de Lausanne, Lausanne, Switzerland accidental conditions than those currently employed. Silicon carbide-based ceramic matrix composites, known as $\mathrm{SiC} / \mathrm{SiC}$, are one the potential replacement material for nuclear fuel cladding in light-water reactors, as well as for structural components in GenIV [1] concepts, such as the gas-cooled fast reactor or the high-temperature reactor.

The experimental method presented here is known as the radial heat flow technique [2-5]. It used to be common practice for testing solid samples until Parker and al. invented the laser-flash method in the 1960s [6]. Nevertheless, it is still currently used to measure the thermal conductivity of fluids, liquids and solid-liquid interfaces. Indeed, recent papers dealing with the thermal analysis of solidifying alloys such as the $\mathrm{Bi}-\mathrm{Ag}$ eutectic system $[7,8]$ or $\mathrm{Sn}-\mathrm{Cd}-\mathrm{Sb}$ ternary systems [9] make use of a radial heat flow apparatus. In these cases, the probed sample - grain boundary grooves - permits the determination of thermal properties such as the Gibbs-Thomson coefficient of these alloys. Further details on these implementations of this method can be found in papers by Karadağ [10], Keslioğlu [11] and Gündüz [12]. In its hereby presented implementation, this method is used with the objective to measure the effective through-thickness thermal conductivity of tubes made of new $\mathrm{SiC} / \mathrm{SiC}$ composite material. Since the laser-flash technique could not be used to measure long hollow tubular samples, a modular, steady-state, experimental apparatus has been built and demonstrated in its ability to assess the thermal conductivity of a reference material.

Since the composite material features a high anisotropy, a measurement in this geometry is highly relevant. In the axial direction, i.e. the tube's $z$-axis, the conductivity is mostly dictated by that of the fibres since those are woven in that direction. On the other hand, the effective thermal conductivity, through the thickness of 
the cladding, is a more complicated issue; it depends on the architecture of the tube, the volume fractions of the respective phases, the residual porosity of the matrix, and the adhesion between the various layers making the whole composite piece. Furthermore, in view of the fact that the designs proposed by the industry [13, 14] are based on complex concentric architectures, understanding the mechanisms of heat transport across such structure is of high importance. As a matter of fact, the radial thermal conductivity of a fuel cladding is a critical parameter, as it influences the fuel centre-line temperature, i.e. reactor operation safety margins.

\section{Theory}

\section{Radial heat flow}

In a radial heat flow apparatus, one measures the temperature drop across a cylindrical sample. Provided that the heat flux across the sample can be determined, it yields a direct measurement of the thermal conductivity. This is different from most of the nowadays commonly used methods such as laser-flash, MDSC or the hot-strip techniques which are all carried out in a transient regime, which are measuring the thermal diffusivity. In most designs, including the one presented here, one ensures that both the circumferential and axial components of the temperature field can be neglected at the measurement location.

Solving the heat equation under those assumptions, in the geometry featured in Fig. 1, yields the radial temperature profiles in the heating element, as well as in the sample. Since the system is given enough time to reach thermal equilibrium, the time derivative is zero. Combining the solution of the heat equation solution with Fourier's conduction law yields Eq. 1.

$$
k=-\frac{1}{T_{\text {in }}-T_{\text {out }}} \frac{\dot{Q}_{\text {in }}}{2 \pi l} \ln \left(\frac{r_{\text {out }}}{r_{\text {in }}}\right) .
$$

With this, the thermal conductivity of a sample of dimensions $r_{\text {in }}, r_{\text {out }}$, and $l$ can be determined, provided that the heat rate through the sample $\dot{Q}_{\text {in }}$ is known. This specific point is discussed in "Heat transfer model" section.

\section{Radiative thermal conductivity}

Depending on the optical properties of the measured material, infrared photons might be able to traverse the sample, thereby contributing to the overall thermal conductivity, as they may carry a non-negligible amount of energy. This subject has been treated by several authors dealing with thermal conductivity measurements of porous ceramics and insulation materials [5, 16, 17]. The radiative or radiation thermal conductivity is a function of the material refractive index $n$, average temperature and the total absorption coefficient $a$.

$k_{\mathrm{rad}}=\frac{16 \sigma n^{2} \bar{T}^{3}}{3 a}$

Although the expression, given in Eq. 2, varies with the third power of the temperature, in the case material with good thermal conductivities and low porosities, it generally contributes only in a limited extent to the effective conductivity of a material, since most conductive materials are either dense or optically opaque in the IR range. On the other hand, both the radiative and gaseous contributions to overall conduction [17] should not be neglected, when a material features high porosity and/or optical properties
Fig. 1 Sketch showing the polar geometry in which the heat equation is solved as well as radial temperature profiles in the heating element and sample, from [15]
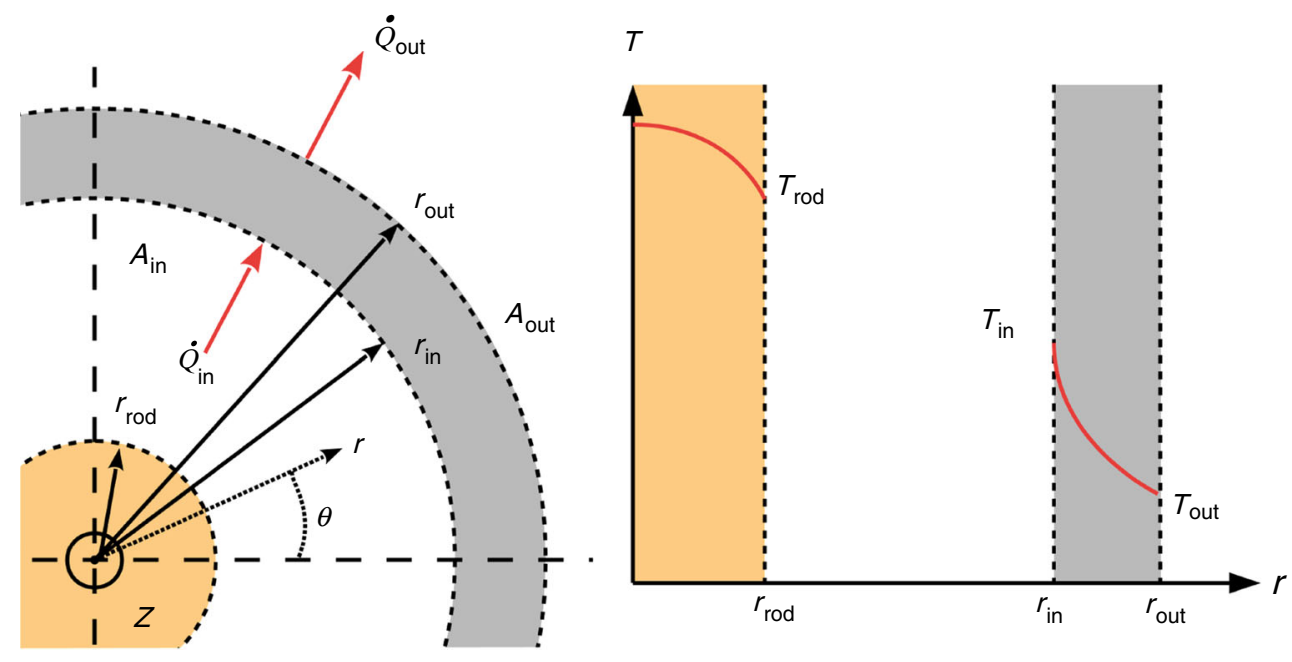
allowing transport infrared radiation. In the present apparatus, the thermal transport through gas molecules is always negligible because the measurements are carried out in vacuum.

\section{Implementation}

The experimental device is made of a 70-cm-high, 20-cmdiameter vacuum chamber, a centrally positioned resistance heating rod made of graphite, a positioning system allowing for samples with diameters varying from 8 to 50 $\mathrm{mm}$ and lengths ranging from 4 to $20 \mathrm{~cm}$. To reduce backreflections of the thermal radiation from the sample to itself via the chamber walls, those have been covered with a carbon-based paint so that their emissivity is closer to that of a black body. A view of the CAD model of the apparatus is shown in Fig. 2.

A GW-Instek PSW 30-108 power supply provides a current ranging from 0 to $108 \mathrm{~A}$ at tensions ranging from 0 to $30 \mathrm{~V}$. Thermocouples are placed at several positions to measure the temperatures required to determine the radiant heat fluxes between the relevant surfaces. In the current implementation, all thermocouples but the one on the heating element are of the $\mathrm{K}$ type. Since this apparatus has been designed to measure over a large range of temperatures, a platinum-rhodium-type $\mathrm{R}$ thermocouple is used on the resistance heater.

Contrarily to designs such as those by Peavy and Flynn $[3,4]$, the sample cannot be kept isothermal along its length with this apparatus. This is due to the limitations imposed on the geometry by aspect ratios of the samples, as well as the need for modularity, so that different cladding designs might be characterised. As a result, additional thermocouples positioned at the top of the sample surface as well as at the top of the resistance heater are necessary to account for the axial temperature distribution along the lengths of the sample and heating rod.

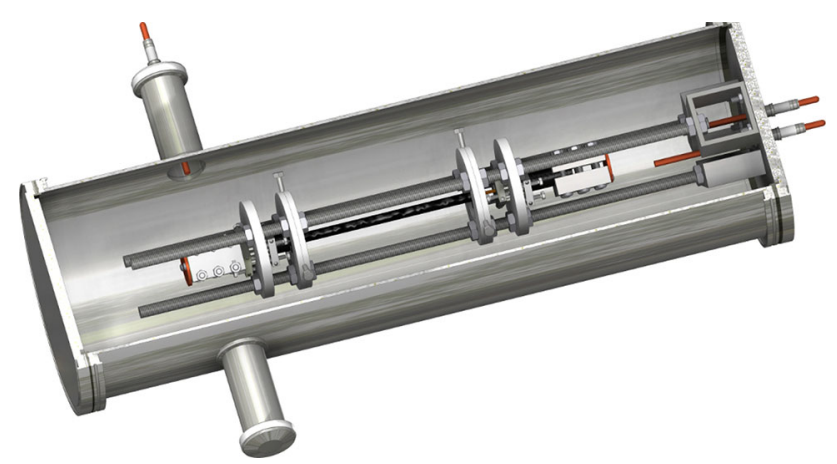

Fig. 2 Rendering of the CAD model created to design the radial heat flow experimental apparatus, from [15]
The shortest possible samples have to be $\sim 30 \mathrm{~mm}$ long, whereas tubes as long as $250 \mathrm{~mm}$ can be fitted in the equipment. In terms of diameters, the smallest inner diameter must be around $5-6 \mathrm{~mm}$ to ensure that the inner thermocouples do not touch the heating rod, since this would lead to asymmetrical heating of the sample. Tubes with diameters as large as $50 \mathrm{~mm}$ can be brought in the apparatus for measurements.

\section{Axial temperature profile}

Even though knowing the axial temperature profile is not required for Eq. 1, which defines the radial thermal conductivity, it is necessary in the determination of the heating rate of the apparatus surfaces. Indeed, losses are likely to occur along the length of the sample, since it is not insulated along the $z$-axis of the sample. As a result, the inner surface of the sample and the heating wire form a "cavity", where the temperature is higher than in the rest of the apparatus. The difference between the measurement points at the top and middle of the heating element can reach magnitudes of more than $500{ }^{\circ} \mathrm{C}$ at high temperatures. This effect has to be taken into account for the correct heat fluxes to be calculated.

The axial component of the temperature profile is described by Bessel-type functions, which are considered through case-specific finite element simulations. In this manner, heat maps such as the one presented in Fig. 3 are obtained. The shape of the $z$-axis profile is then extracted from these and fitted using Fourier series such as Eq. 3.

$$
T(z)=a_{0}+\sum_{i} a_{\mathrm{i}} \cos (i \omega z)+b_{\mathrm{i}} \sin (i \omega z)
$$

Figure 3 illustrates the reason why the temperatures of the sample and of the heating element cannot be assumed to be uniform. Moreover, since radiosity calculations require a single temperature to be defined for each of the surfaces, the average of the three profiles shown in Fig. 3 should be used.

\section{Heat transfer model}

A drawback of this implementation of the radial heat flow method is that the measurements have to be carried out in vacuum to avoid oxidation, meaning that only thermal radiation ensures transfer of heat between the bodies. Therefore, an analysis of the radiant heat fluxes at a selection of relevant surfaces is required.

The sketch presented in Fig. 4 shows all the locations, at which temperatures are recorded. This sketch does not respect the actual proportions (see Fig. 2 for this), but illustrates the simplified geometry used in the upcoming 


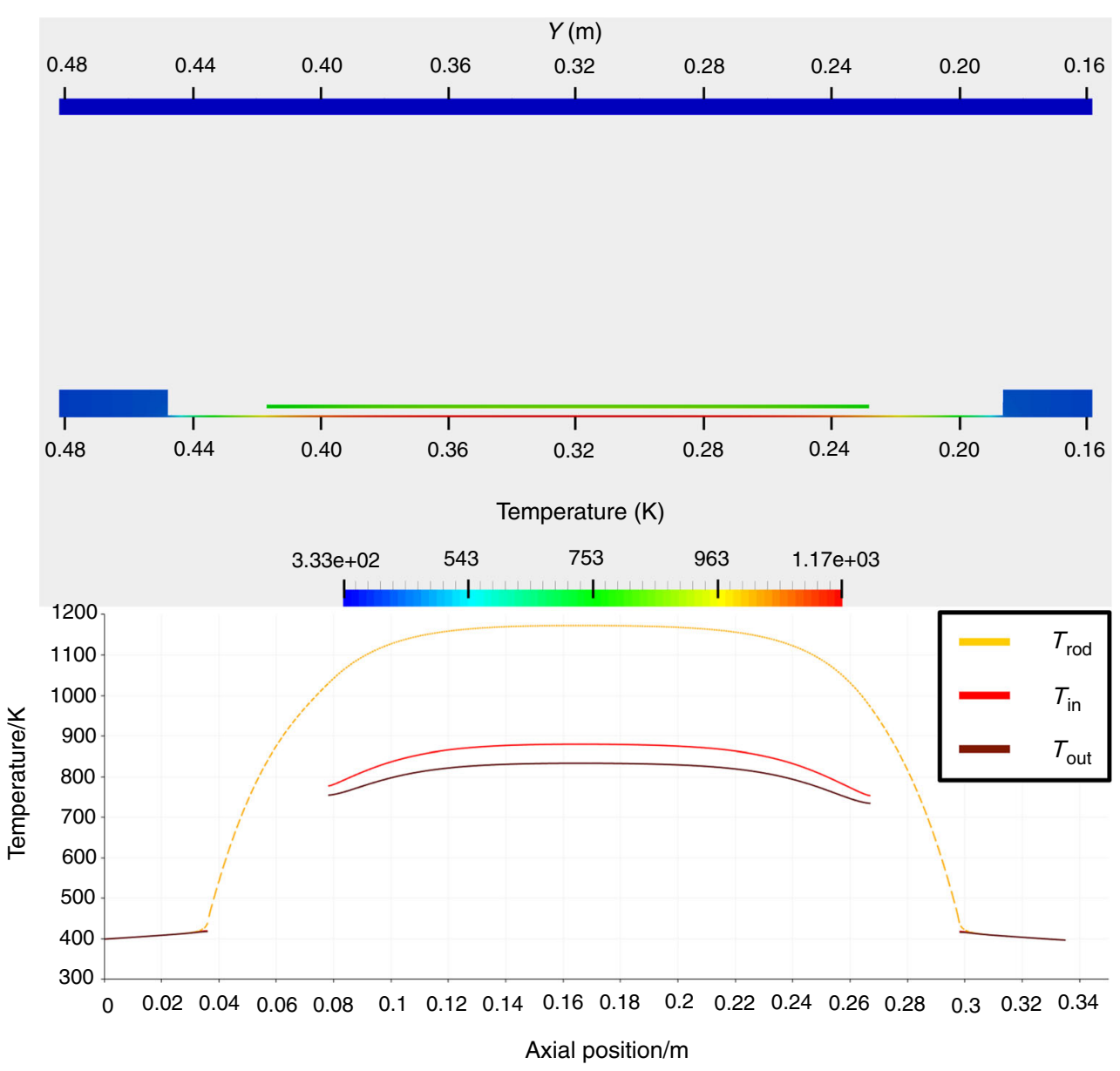

Fig. 3 FEM heat map output showing the axial temperature gradients along both the sample and heating wire surfaces (top) and associated temperature profiles (bottom)

analysis. Both larger cylinders placed at the ends of the heating element serve as electrical connections, as well as holders. These do not need considering in the later discussion because four $\mathrm{ZrO}_{2}$ discs are used to maintain the sample and the cylinders at their $z$-axis positions. Thanks to these, the components either above or below the discs are shielded from thermal radiation in the axial direction. For this reason, a subset of six surfaces can be considered in the radiant heat transfer calculations. This set is schematically represented in Fig. 5. The parameters $F_{\mathrm{ij}}$ are the view factors between any pair of surfaces $i$ and $j$. These are geometrical functions describing the extent to which a surface $i$ has a direct line of sight over another surface $j$.

\section{Radiosity analysis}

The radiosity method is a well-established analytical scheme used to calculate the radiant heat transfer among surfaces forming an enclosure [18].
$J_{\mathrm{e}, \mathrm{i}}=\frac{\mathrm{d} \Phi}{\mathrm{d} A}=J_{\mathrm{e}, \mathrm{em}}+J_{\mathrm{e}, \mathrm{r}}+J_{\mathrm{e}, \mathrm{tr}}$

In Eq. 4, the subscripts $e m, r$ and $t r$, respectively, stand for the emitted, reflected and transmitted parts of the radiant heat flux per unit area $\Phi$. Since all the bodies used in the apparatus are opaque, the transmitted parts are zero, leaving only two parts to determine.

The method used to analyse the heat exchange is the formalism developed by Gebhart [19]. Gebhart's approach determines the factors $\Gamma_{\mathrm{ij}}$, which account for the fraction of the energy leaving a surface $i$, reaching another one, noted $j$, and being absorbed there. The starting point of the analysis is the energy balance on any surface $j$ :

$Q_{\mathrm{j}}=A_{\mathrm{j}} \varepsilon_{\mathrm{j}} \sigma T_{\mathrm{j}}^{4}-\sum_{i=1}^{N} \Gamma_{\mathrm{ij}} A_{\mathrm{i}} \varepsilon_{\mathrm{i}} \sigma T_{\mathrm{i}}^{4}$

Here, the first term is the thermal radiation emitted by the surface, whilst the second is the sum of the energies radiated by the other surfaces absorbed at $j$. The factors $\Gamma_{\mathrm{ij}}$ 


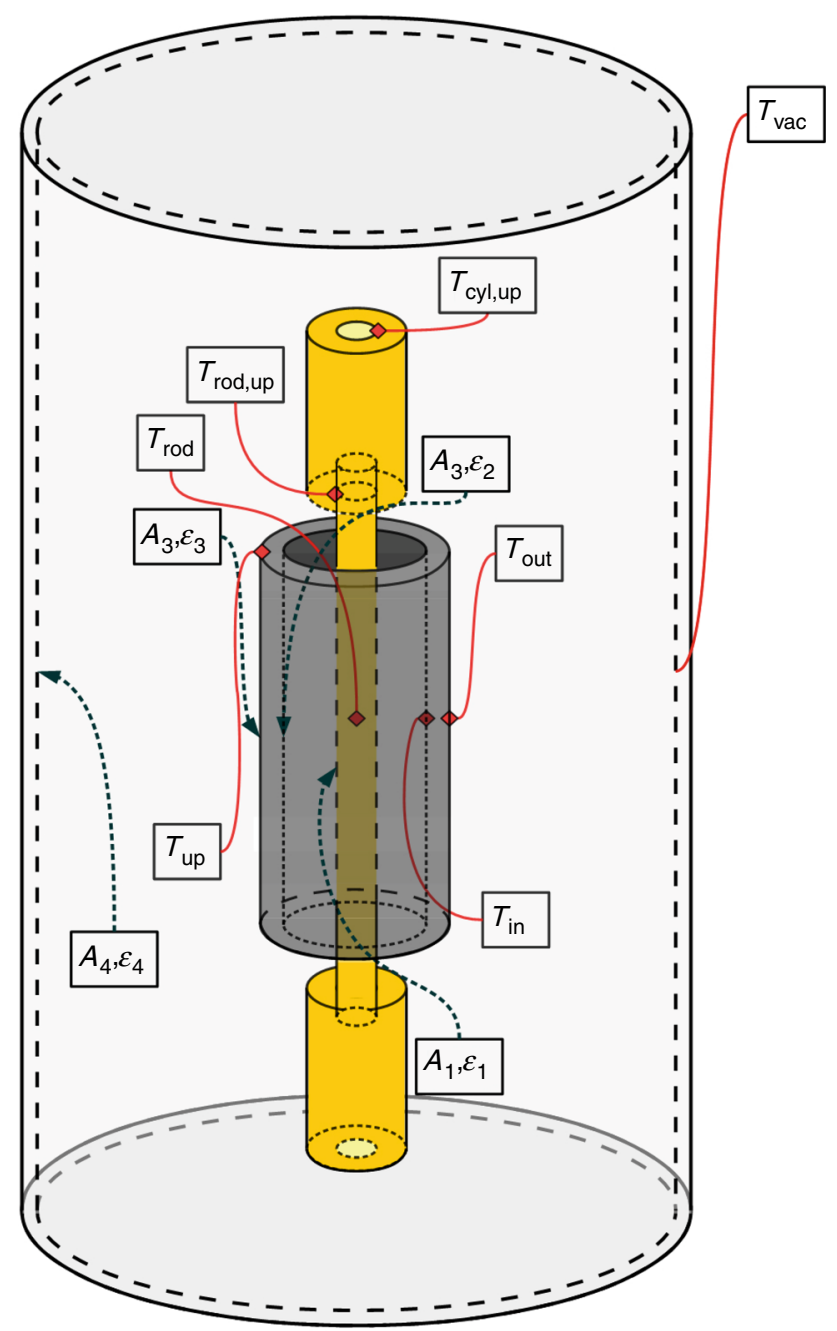

Fig. 4 Schematic drawing showing the points at which temperatures are measured. The drawing is not at a 1:1 scale, from [15]

include all possible paths allowing thermal radiation to go from $i$ to $j$, which means that even though $i$ and $j$ might not share a direct line of sight, $\Gamma_{\mathrm{ij}}$ is not necessarily zero. Therefore, the amount of energy leaving $i$ and absorbed at $j$ is described by Eq. 6 .

$\Gamma_{\mathrm{ij}} E_{\mathrm{i}}=E_{\mathrm{i}}\left(\varepsilon_{\mathrm{j}} F_{\mathrm{ij}}+\sum_{k=1}^{N} F_{\mathrm{i}, \mathrm{k}} \rho_{\mathrm{k}} \Gamma_{\mathrm{k}, \mathrm{j}}\right)$

After some algebraic manipulation, this expression is expanded on the indices $i=1, N$ and $j=1, N$, thus obtaining what is known as the Gebhart matrix $\Gamma$.
The matrix is obtained by solving $\Gamma=\boldsymbol{M}^{-1} \boldsymbol{F}$. The factors of this matrix have properties similar to view factors:

- $\sum_{\mathrm{i}=1}^{N} \Gamma_{\mathrm{ij}}=1$ since all the energy leaving a given surface has to be absorbed somewhere in the enclosure.

- $\varepsilon_{\mathrm{i}} A_{\mathrm{i}} \Gamma_{\mathrm{ij}}=\varepsilon_{\mathrm{j}} A_{\mathrm{j}} \Gamma_{\mathrm{ji}}$, known as the reciprocity rule, which directly follows from the definition of view factors.

Introducing the Gebhart factors calculated with Eq. 7 in the energy balance from Eq. 5 yields the following expression for the heat rate at a given surface of the enclosure:

$\dot{Q}_{\mathrm{i}}=A_{\mathrm{i}} \varepsilon_{\mathrm{i}} \sum_{\mathrm{j}=1}^{N} \Gamma_{\mathrm{ij}} \sigma\left(T_{\mathrm{i}}^{4}-T_{\mathrm{j}}^{4}\right)$

Using this expression, the amount of heat going through the sample, $\dot{Q}_{\text {in }}$, required in Eq. 1 is found by taking the difference between the heat rate exiting the sample outer surface, $\dot{Q}_{5}$, and that entering the inner wall of the tube, $\dot{Q}_{4}$. Note that the indices 4 and 5 refer to the ones sketched in Fig. 5.

\section{View factors}

Based on the sketch featured in Fig. 5, not all of the 36 possible view factors are greater than zero. As a matter of fact, exploiting some basic properties of view factors, several of these have to be zero. A summary of the factors required to describe the heat exchange are gathered in Table 1.

The analytical expressions used to calculate the factors $F_{41}, F_{44}$, and $F_{\mathrm{i} 6}, \quad i=2,3,5$ can be found in papers by Brockmann, Leuenberger and Person as well as Rea [20-22].

\section{Results}

\section{Model validation}

A 20-cm-long tube of stainless steel (316L), with an inner diameter of $38.4 \mathrm{~mm}$ and a wall thickness of $2 \mathrm{~mm}$, was used to validate the heat transfer model. $316 \mathrm{~L}$ was chosen because of its availability, and the easiness to weld thermocouples on a tube made with this alloy. Additionally, the conductivity of this steel is well documented, with full temperature range coverage data available. Based on data

$$
\overbrace{\left(\begin{array}{ccc}
1-\rho_{1} F_{11} & \cdots & -\rho_{N} F_{1 N} \\
\vdots & \ddots & \vdots \\
-\rho_{1} F_{N 1} & \cdots & 1-\rho_{N} F_{N N}
\end{array}\right)}^{\boldsymbol{M}} \overbrace{\left(\begin{array}{ccc}
1-\rho_{1} F_{11} & \cdots & -\rho_{N} F_{1 N} \\
\vdots & \ddots & \vdots \\
-\rho_{1} F_{N 1} & \cdots & 1-\rho_{N} F_{N N}
\end{array}\right)}^{\Gamma}=\overbrace{\left(\begin{array}{ccc}
1-\rho_{1} F_{11} & \cdots & -\rho_{N} F_{1 N} \\
\vdots & \ddots & \vdots \\
-\rho_{1} F_{N 1} & \cdots & 1-\rho_{N} F_{N N}
\end{array}\right)}^{F}
$$




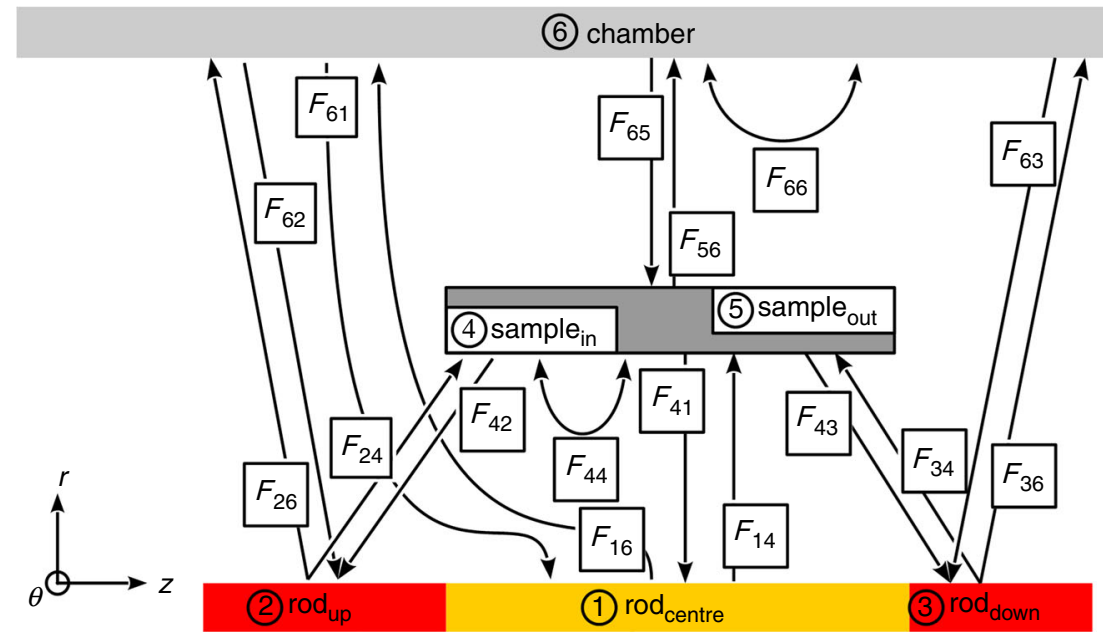

Fig. 5 View in the $(r, z)$ plane of the surfaces considered to determine the radiant heat exchange in the apparatus, from [15]

Table 1 Summary of the view factors implicated in the radiosity calculations determining the heat fluxes between the heating rod, the sample and the vacuum chamber

\begin{tabular}{lllllll}
\hline$i i^{j}$ & $F_{\mathrm{i}, 1}$ & $F_{\mathrm{i}, 2}$ & $F_{\mathrm{i}, 3}$ & $F_{\mathrm{i}, 4}$ & $F_{\mathrm{i}, 5}$ & $F_{\mathrm{i}, 6}$ \\
\hline$F_{1, \mathrm{j}}$ & $\mathbf{0}$ & 0 & 0 & $F_{14}$ & 0 & $\mathbf{F}_{\mathbf{1 6}}$ \\
$F_{2, \mathrm{j}}$ & 0 & $\mathbf{0}$ & 0 & $\mathbf{F}_{\mathbf{2 4}}$ & 0 & $F_{26}$ \\
$F_{3, \mathrm{j}}$ & 0 & 0 & $\mathbf{0}$ & $\mathbf{F}_{\mathbf{3 4}}$ & 0 & $F_{36}$ \\
$F_{4, \mathrm{j}}$ & $F_{41}$ & $F_{42}$ & $F_{43}$ & $F_{44}$ & 0 & 0 \\
$F_{5, \mathrm{j}}$ & 0 & 0 & 0 & 0 & $\mathbf{0}$ & $F_{56}$ \\
$F_{6, \mathrm{j}}$ & $F_{61}$ & $F_{62}$ & $F_{63}$ & $F_{64}$ & $F_{65}$ & $\mathbf{F}_{\mathbf{6 6}}$ \\
\hline
\end{tabular}

Surface indices are the same as the ones used in Fig. 5. The emphasis code is the following: italic $\equiv$ equation from literature, bold $\equiv$ sum rule, without emphasis $\equiv$ reciprocity, regular " 0 " $\equiv$ no line of sight and bold " 0 " $\equiv$ flat or convex surfaces

published by Ho et al. [23], Eq. 9 is used to fit the thermal conductivity of $316 \mathrm{~L}$ for temperatures between the ambient and $1000{ }^{\circ} \mathrm{C}$.

$k(T)=6.321+0.09147 \cdot T^{0.7631}$
Figure 6 shows the data acquired in a typical experimental run, the first graph features the recorded temperatures, whilst the second shows the emitted and absorbed heating rates at the considered surfaces. It shall be noted, that the temperatures measured on the sample, $T_{\text {in }}, T_{\text {out }}$ and $T_{\text {up }}$, are almost identical at temperatures below $100^{\circ} \mathrm{C}$.

The data displayed in Fig. 7 shows experimental data with the fit of $316 \mathrm{~L}$ literature data. The curve does not reach high temperatures, because the radiative heat transfer between the heating rod and the sample is inefficient. Indeed, since the emissivity of the steel tube is rather low, it does not absorb much energy. Moreover, the lower part of the temperature range, below an average sample temperature of $100{ }^{\circ} \mathrm{C}$ does not fit the data from [23]. This is because the power required to heat up the sample in this range are extremely small, resulting in gradients too close to the accuracy of the thermocouples. Significant cooling of the external surface of the vacuum chamber could be implemented to reduce this effect by increasing the power required to reach equilibrium, thus possibly enabling measurements down to the ambient.

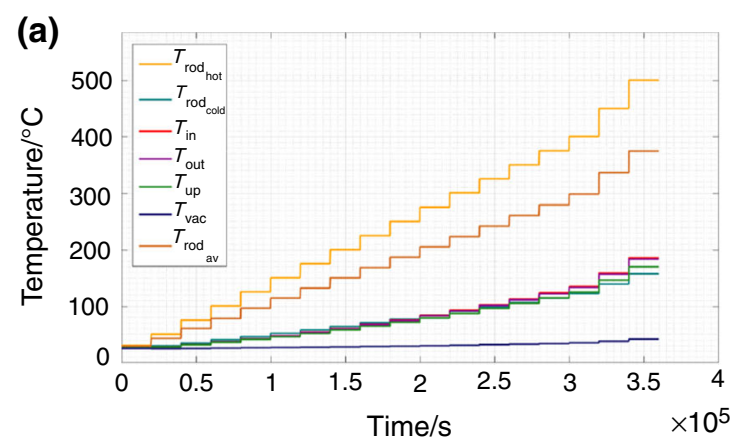

(b)

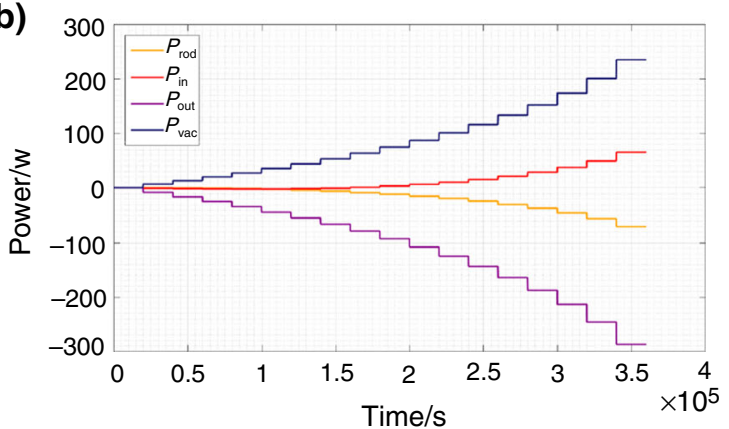

Fig. 6 Recorded temperatures $\mathbf{a}$ as a function of measurement time and power $\mathbf{b}$ absorbed (positive values) and emitted (negative values) by the surfaces relevant to the modelling of the heat transfer in the experimental apparatus. a Temperatures. b Heating rates 


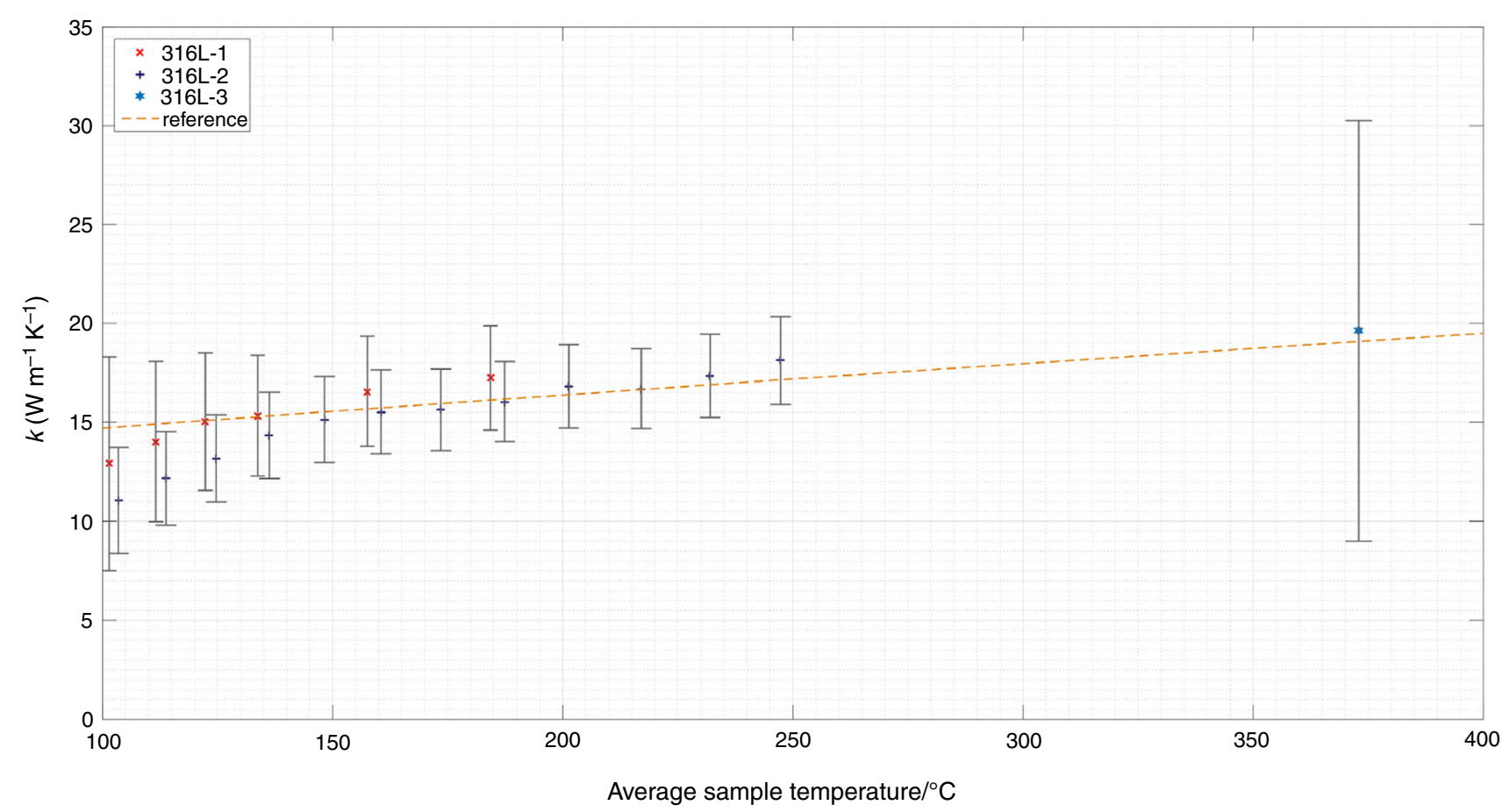

Fig. 7 Measured thermal conductivity of a 316L tube against fitted historical data from Ho et al. [23]

\section{$\mathrm{SiC} / \mathrm{SiC}$ measurements}

Figure 8 shows experimental results from measurements carried out on $\mathrm{SiC} / \mathrm{SiC}$ tubes provided by General Atomics. A cross-sectional view of this material is shown in the vignette displayed on the upper left corner. The results show that a simpler structure, featuring less interfaces between different types of materials, are desirable. Indeed, the sample GA-1 shows the highest measured conductivities, reaching about $3-4 \mathrm{~W} \mathrm{~m}^{-1} \mathrm{~K}^{-1}$ thanks to the absence of an interface between monolithic $\mathrm{SiC}$ and $\mathrm{SiC} / \mathrm{SiC}$ layers such as the ones visible on the

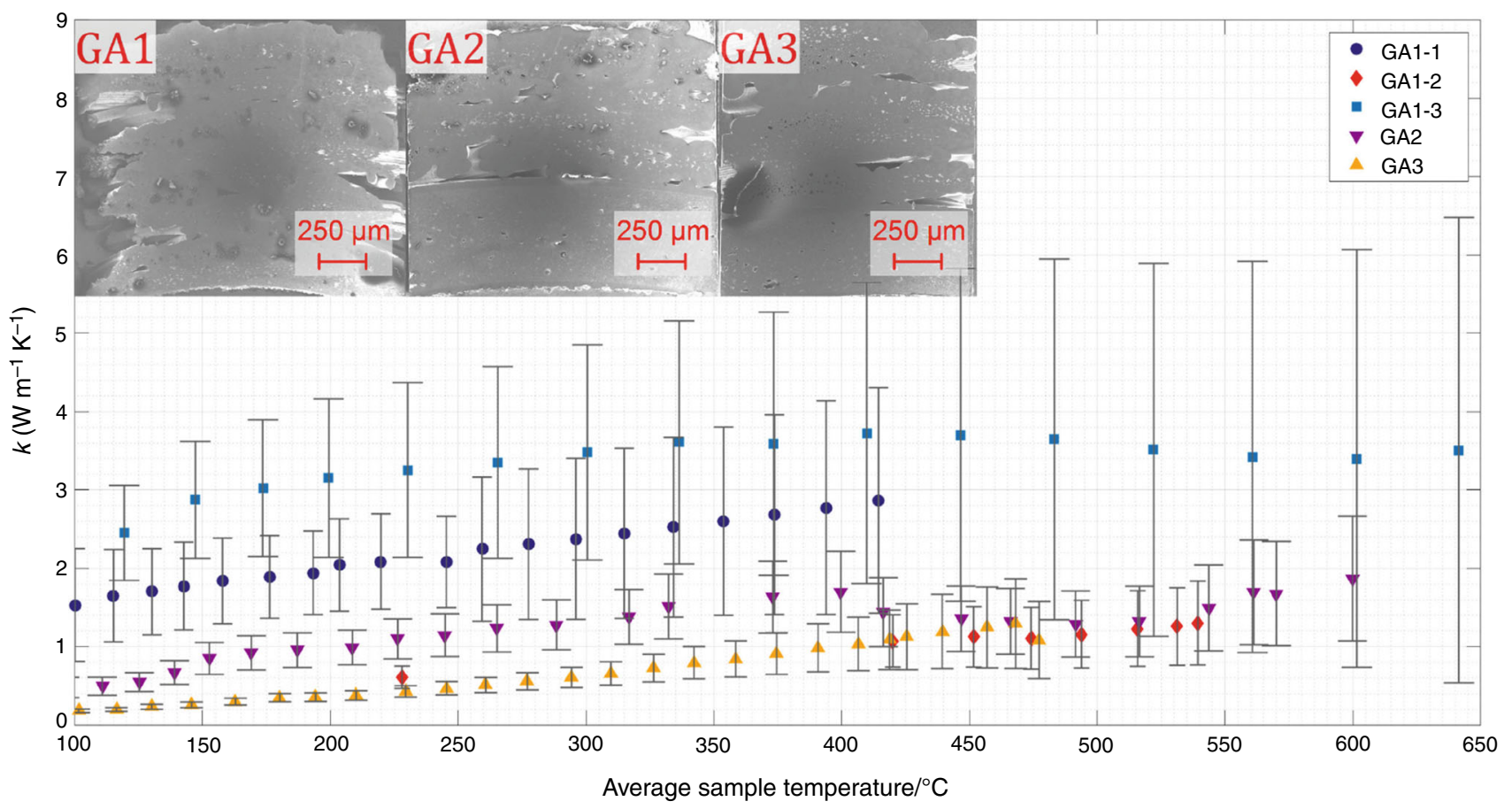

Fig. 8 Thermal conductivity measured on a set of general atomics $\mathrm{SiC} / \mathrm{SiC}$ composite tubes 
other two cross sections shown in Fig. 8. Compared to measurements performed on $\mathrm{SiC} / \mathrm{SiC}$ platelets such as those reported by Youngblood [24] or Kohyama [25, 26], who measured conductivities of $5-15 \mathrm{~W} \mathrm{~m}^{-1} \mathrm{~K}^{-1}$, this new data shows that the prototype composite tubes are less conductive than lab-scale produced planar samples. In addition to significant geometrical and structural differences, the radial heat flow apparatus presented here separates the anisotropic components of the thermal conductivity, thus addresses only the lower transverse part of the effective conductivity. Furthermore, in the case of more porous structures, such as those of the GA-2 and GA-3 samples, photonic heat transport contributes to the overall conductivity since these become more conductive as the temperature increases.

The reason behind the very low thermal conductivity of these assemblies is attributed to their microstructure. Since it features several layers, which attachment to one another is rather poor, the thermal resistances at each of the interfaces is extremely high. This results in an insulating behaviour at low temperatures. In addition to this, the inhomogeneously distributed large pores lead to scatter in the thermal conductivity. As a matter of fact, depending on the measurement location, the conductivity of these tubes can almost vary by an order of magnitude. As the temperature elevates to a point where thermal radiation within the material becomes sufficient to contribute to the overall conductivity, the material shows a slight improvement in its heat transfer ability. Nonetheless, the behaviour of the here measured samples is not satisfactory for its intended use as a nuclear cladding. However, new materials candidates have already been developed by General Atomics, which show a much enhanced microstructure and layer bonding. According to measurements by the supplier, these samples also show a better heat transfer [27].

\section{Conclusions}

This paper presents a new implementation of a classical thermal conductivity measurement technique. Since the development of the laser-flash method in the sixties [6], steady-state measurements such as the radial heat flow have fallen in disuse. This apparatus was successfully tested and validated using a 316L stainless steel reference. In this article, it is shown that in specific cases, a direct measurement method can be used, and even be preferable. Highly anisotropic $\mathrm{SiC} / \mathrm{SiC}$ composite tubes, which properties are influenced by their implementation geometry, are such a case. The advantage of this experimental device is that the actual transverse conductivity is obtained, whereas laser-flash measurements usually yield a response in which both the transverse and axial conductivities are partially mixed. More importantly, it enables the measurement of the properties of prototypes in their real design, instead of small platelets. Such a comprehensive testing is mandatory to assess the real quality of the final assembly, intended for an application where safety requirements are so high that no error can be allowed.

The experimental results highlight the necessity for good adhesion between layers made of different materials in complex systems such as multilayered $\mathrm{SiC} / \mathrm{SiC}$ composite tubes. Lastly, this apparatus could easily be adapted to perform measurements on other types of samples and materials. It could for instance be fitted with a hollow cylindrical cell, with which the very actual topic of nanofluids could be addressed [28, 29].

Acknowledgements The authors would like to acknowledge the CARAT (DE-NE0000566), GIF VHTR, CARAT (DE-NE0000566), EU Fp7 MaTISSE project (EU Grant No. 604862) and MeAWaT (ccem.ch/meawat) projects for funding this work.

\section{References}

1. GIF Portal-Home-Public. https://www.gen-4.org/gif/jcms/c_ 9260/public.

2. Czichos H, Saito T, Smith L, editors. Springer handbook of materials measurement methods. Berlin: Springer; 2006.

3. Peavy BA. Steady state heat conduction in cylinders with multiple continuous line heat sources. J Res Natl Bur Stand C Eng Instrum. 1963;67C(2):119-28.

4. Flynn DR. A radial-flow apparatus for determining the thermal conductivity of loose-fill insulations to high temperatures. J Res Natl Bur Stand C Eng Instrum. 1963;67C(2):129-37.

5. Lezhenin FF, Shurgay PI, Kostornov AG, Shevchuk MS. Effective thermal conductivity in the $373-1400{ }^{\circ} \mathrm{K}$ temperature range. Heat Transf Sov Res. 1977;9(2):144-50.

6. Parker WJ, Jenkins RJ, Butler CP, Abbott GL. Flash method of determining thermal diffusivity, heat capacity, and thermal conductivity. J Appl Phys. 1961;32(9):1679-84.

7. Farahany S, Idris MH, Ourdjini A, Faris F, Ghandvar H. Evaluation of the effect of grain refiners on the solidification characteristics of an Sr-modified ADC12 die-casting alloy by cooling curve thermal analysis. $\mathrm{J}$ Therm Anal Calorim. 2015;119(3):1593-601.

8. Altıntas Y, Öztürk E, Aksöz S, Keşlioğlu K, Maraşlı N. Thermal conductivity and interfacial energy of solid $\mathrm{Bi}$ in the $\mathrm{Bi}-\mathrm{Ag}$ eutectic system. J Therm Anal Calorim. 2015;122(1):65-72.

9. Öztürk E, Aksöz S, Altıntas Y, Keşlioğlu K, Maraşlı N. Experimental measurements of some thermophysical properties of solid $\mathrm{CdSb}$ intermetallic in the $\mathrm{Sn}-\mathrm{Cd}-\mathrm{Sb}$ ternary alloy. J Therm Anal Calorim. 2016;126(3):1059-65.

10. Karadă̆ SB, Altıntas Y, Öztürk E, Aksöz S, Keşlioğlu K, Maraşlı N. Solid-liquid interfacial energy of solid succinonitrile solution in equilibrium with succinonitrile-neopentylglycol eutectic liquid. J Cryst Growth. 2013;380:209-17.

11. Keşlığlu K, Gündüz M, Kaya H, Çadırlı E. Solid-liquid interfacial energy in the $\mathrm{Al}-\mathrm{Ti}$ system. Mater Lett. 2004;58(24):3067-73. 
12. Gündüz M, Hunt JD. The measurement of solid-liquid surface energies in the $\mathrm{Al}-\mathrm{Cu}, \mathrm{Al}-\mathrm{Si}$ and $\mathrm{Pb}-\mathrm{Sn}$ systems. Acta Metall. 1985;33(9):1651-72.

13. Maxime Z, David P, Ravenet A, Rochais D. Commissariat A L'energie atomique et aux energies alternatives, assignee. Solid interface joint with open porosity, for nuclear fuel rod. WO/2011/ 157780; 2011.

14. Composite cladding for nuclear fuel rods. US 5790623 A; 1998.

15. Fave L. Investigation of the thermal conductivity of $\mathrm{SiC} / \mathrm{SiC}$ cladding before and after irradiation. Lausanne: École polytechnique fédérale de Lausanne; 2017.

16. Zhu D, Spuckler CM. The lattice and thermal radiation conductivity of thermal barrier coatings. Daytona Beach: Magazines \& Publications; 2008.

17. Flynn DR. Thermal conductivity of ceramics. In: Wachtman JB, Society AC, American Society for Testing and Materials, National Bureau of Standards, editors. Proceedings of mechanical and thermal properties of ceramics; 1969. p. 63-123.

18. Howell JR, Mengüç MP, Siegel R. Thermal radiation heat transfer. 6th ed. Boca Raton: CRC Press; 2015.

19. Gebhart B. Surface temperature calculations in radiant surroundings of arbitrary complexity for gray, diffuse radiation. Int $\mathrm{J}$ Heat Mass Transf. 1961;3(4):341-6.

20. Rea SN. Rapid method for determining concentric cylinder radiation view factors. AIAA J. 1975;13(8):1122-3.

21. Brockmann H. Analytic angle factors for the radiant interchange among the surface elements of two concentric cylinders. In J Heat Mass Transf. 1994;37(7):1095-100.
22. Leuenberger H, Person RA. Compilation of radiation shape factors for cylindrical assemblies. New York: American Society of Mechanical Engineers; 1956.

23. Ho CY, Ackerman MW, Bogaard RH, Chu TK, Desai PD. Thermal conductivity and thermal diffusivity of selected carbon steels, chromium steels, nickel steels, and stainless steels. New York: Springer; 1978.

24. Youngblood GE, Senor DJ, Jones RH, Kowbel W. Optimizing the transverse thermal conductivity of $2 \mathrm{D}-\mathrm{SiCf}, \mathrm{SiC}$ composites, II. Experimental. J Nucl Mater. 2002;307:1112-9.

25. Kowbel W, Bruce CA, Tsou KL, Patel K, Withers JC, Youngblood GE. SiC thermal conductivity, composites $\mathrm{SiC}$, for fusion applications. J Nucl Mater. 2000;238:128-37.

26. Kohyama A. CMC for nuclear applications. In: Krenkel IW, editor. Ceramic matrix composites. New York: Wiley; 2008. p. $353-84$.

27. Zhang J, Khalifa HE, Deck C, Sheeder J, Back CA. Thermal diffusivity measurement of curved samples using the flash method. In: Maty J, Katoh Y, Lin HT, Vomiero A, editors. Ceramic engineering and science proceedings. Hoboken: Wiley; 2015. p. 43-56.

28. Paul G, Chopkar M, Manna I, Das PK. Techniques for measuring the thermal conductivity of nanofluids: a review. Renew Sustain Energy Rev. 2010;14(7):1913-24.

29. Bashirnezhad K, Rashidi MM, Yang Z, Bazri S, Yan WM. A comprehensive review of last experimental studies on thermal conductivity of nanofluids. $\mathrm{J}$ Therm Anal Calorim. 2015;122(2):863-84. 